\title{
Accuracy of Discriminating between Similar Drug Names by Nurses: Effect of Finger-pointing on Error Prevention
}

\author{
Junko Mitobe \& Takahiro Higuchi \\ Graduate School of Human Health Sciences, Tokyo Metropolitan University \\ 1-1, Minami-Osawa, Hachioji, Tokyo, 192-0397, Japan \\ Tel. +81-42-677-1111 E-mail: mitobe-junko@hs.tmu.ac.jp
}

Received: August 23, 2017 Accepted: December 20, 2017 Published: December 22, 2017

doi:10.5296/jss.v3i1.11732 URL: https://doi.org/10.5296/jss.v3i1.11732

\begin{abstract}
Discriminating between similar drug names accurately is important in order to prevent medication error. To facilitate accurate discrimination, performing finger-pointing toward drug names to recognize them is recommended in Japan for healthcare workers. We investigate whether nurses would accurately recognize the difference between target and similar drug names and whether finger-pointing would lead to error prevention for drug names by using a choice reaction time task. Participants observed six drug names with or without pointing with the index finger and determined as quickly and accurately as possible whether the target drug name was present. Targets were real drug names, although distractors were pseudo names so as to strictly manipulate the degree of similarity. The results showed that error rates were significantly higher for nurses than for students. Due to their familiarity with the drug names, the nurses could misrecognize the pseudo words as target drug names when a quick response was requested. We also found that nurses did not receive a benefit from finger-pointing. Moreover, finger-pointing may have been inadequate to lead to accurate recognition of complex stimuli, such as drug names.
\end{abstract}

Keywords: drug names, medication error, expertise, nurse, error prevention, finger-pointing

\section{Introduction}

Prevention of medical errors is an important issue in protecting patients' health and avoiding great cost to hospitals. A common medical error is medication error, such as medication order, prescribing, or administration errors (Kawamura, 2003; Kohn, Corrigan, \& Donaldson, 1999; Pham et al., 2012; Japan Council for Quality Health Care Division of Adverse Event 
Prevention, 2016). For prevention of a medication error, it has been strongly recommended that healthcare workers use finger-pointing toward drugs and prescriptions when they prepare and administer medicine to patients. Finger-pointing is recommended while reading aloud the indicated object (called finger-pointing and calling). Several studies showed evidence that finger-pointing has the potential to prevent errors. For example, finger-pointing leads participants to fix their gaze longer and focus their attention on the object that they should confirm (Shinohara, Morimoto, \& Kubota, 2009; Masuda, Shigemori, Sato, \& Haga, 2014). So far, the evidence of finger-pointing benefit is limited in that there are no studies testing healthcare workers to investigate the effect of finger-pointing. The present study was therefore designed to test nurses' ability to accurately discriminate between similar drug names (i.e., the ability to avoid confusion errors) with or without finger-pointing. Because the study was conducted in Japan, the confusion errors could be related at least in part to orthographic and phonetic characteristics of the Japanese language.

\subsection{Medication errors due to similarity of drug names}

The Japan Council for Quality Health Care (2016) reported that over 30 percent of medical incidents in Japanese hospitals are related to medication errors. Previous studies showed that both orthographically (look alike) and phonetically (sound alike) similar drug names are likely to lead to medication errors (Lambert, Chan, \& Lin, 2001, 2003; Lambert, Chan, \& Gupta, 2003; Berman, 2004). Most Japanese drug names are written in Katakana, which consists of phonetic symbols and is often used to transcribe a loan word. Previous studies suggested that medication errors in Japan seem to be related to the similarity of drug names written in Katakana (Suzuki et al, 2005; Nabeta, Imai, Kimura, Ohkura, \& Tsuchiya, 2011).

To quantify the similarity of drug names written in Katakana, Tsuchiya, Kawamura, Oh, and Hara (2001) created "the index of similarity between drug names." This index includes three representative sub-indices called "cos," "htco," and "edit." The cos index expresses the number of same letters used between the names. The htco index indicates whether the same letter was used for the beginning and the end of the two names. The edit index means the number of manipulations for names (i.e. substituted, transposed, inserted, or discarded letters) so that two names become coincident. Yamade, Haga, Tsuchiya, and Shin (2006) tested both pharmacists (i.e., those with specific knowledge about medication) and university students (i.e., those with no specific knowledge about medication) and demonstrated that, for both groups of participants, confusion errors for similar drug names increased with the degree of cos and edit indices for the names. This suggests that confusion errors are likely to occur more frequently when drug names share letters at the beginning and/or the end of syllables.

\subsection{The relation between expertise and accuracy}

There are contradictory findings with regard to the relationship between expertise in medication and accuracy in discriminating between similar drug names. Some studies showed that experts in medication are better at discriminating between similar drug names. Lambert et al. (2003) showed using a visual identification task with drug names that pharmacists identified drug names more accurately than did university students. Lambert et al. interpreted the results based on the interactive activation model of word perception. The interactive 
activation model was proposed by McClelland and Rumelhart (McClelland \& Rumelhart, 1981; Rumelhart \& McClelland, 1982). This model consisted of three levels of hierarchy: feature, letter and word. Feature-based processing is recognized as lower-level processing, whereas word-based processing is recognized as higher-level processing. Based on the model, Lambert et al. explained that pharmacists would show superior ability to identify drug names because, due to their knowledge about medicine and daily experience in handling it, they have established word- based processing for the drug names. In contrast, university students, who are not familiar with drug names, may have used letter-based processing.

In contrast to these studies, several others indirectly suggest that specialized knowledge of drug names can interfere with accurate recognition of the names. O'Connor and Forster (1981) showed that it was more difficult to detect spelling mistakes $t$ in high-frequency words than in low-frequency words. Perea and Lupker (2003) also showed that transposed-letter non-words and substituted-letter non-words can be easily misperceived as words. Considering these findings, discrimination between similar drug names can be particularly difficult for medication experts due to their frequent handling of the drugs.

\subsection{Finger-pointing and calling}

To avoid medication errors, healthcare workers in Japan have been advised to use finger-pointing while reading aloud drugs names and prescriptions when they prepare and administer medicine to patients. Finger-pointing and calling were originally used by train drivers when they checked meters, signals, and signs while driving. Haga, Akatsuka, and Shirato (1996) tested the effects of finger-pointing and calling in an experimental setting using a multi-choice reaction task. In this task, participants reacted as accurately and quickly as possible to suitable color stimuli shown with a "traffic-light-like" background stimulus. Comparisons of accuracy in the task among four conditions (finger-pointing and calling, finger-pointing, calling, and control conditions) showed that the error rate was significantly lower under the finger-pointing and calling and finger-pointing conditions than under the control condition. Based on these findings, finger-pointing and calling has been recommended as a method of avoiding confusion errors for various practical fields, including manufacturing, nuclear industries and healthcare settings.

The positive effect of finger-pointing and calling (particularly the effect produced by finger-pointing) has been explained on the basis of the relation of these actions to attention and gaze direction. In Shinohara et al. (2009), participants performed a Posner's pre-cueing task (Posner, 1980) to investigate the effects of orienting attention with finger-pointing and calling. The results showed that the reaction time (RT) from presenting the target to pressing the key was significantly shorter under the finger-pointing and calling condition and finger-pointing only condition than under the control condition. This finding suggests that finger-pointing has the potential to direct one's attention to the pointed object. The effects of finger-pointing can also be explained on the basis of eye movement. The fixation toward the first target was maintained until pointing toward the first target was terminated when participants were instructed to point their finger to two targets consecutively (Carey, 2000; Neggers \& Bekkering, 2000, 2001, 2002; Deconinck, Polanen, Savelsbergh \& Bennett, 2011). 
For this effect, those who are conducting finger-pointing would be able to fixate on the object pointed to for a relatively long time with central vision, which could lead to accurate recognition.

Recently, Mitobe and Higuchi (2016) suggested that finger-pointing anchors people's eyes on each drug name and prevents error. Participants who were graduate or undergraduate university students observed four drug names with or without pointing with the index finger and determined as quickly and accurately as possible whether the target drug name was present. The results showed that finger-pointing was effective to reduce the error rate when only a single similar drug name existed in the choices. Analyses of gaze behavior showed that the fixation duration and number of fixations per drug name increased under the finger-pointing condition. This suggests that finger-pointing may lead to observing the name more carefully and, consequently, preventing confusion between similar drug names.

Although Mitobe and Higuchi (2016) showed the positive effect of finger-pointing on preventing confusion between similar drug names, only students, who are unfamiliar with drug names, were tested. Therefore, it remains unknown whether healthcare workers, who are very familiar with drug names, would also receive a benefit from finger-pointing. Testing this is particularly important, since those who are familiar with the drug names could confuse similar names due to the top-down process of words (i.e., priming) (O'Connor \& Forster ,1981; Perea \&Lupker, 2003).

The purpose of this study was to investigate (a) whether nurses, one type of the expert in medication, would be able to recognize similar drug names accurately when rapid reaction is requested and (b) whether nurses would receive a benefit from finger-pointing. In the present study, we focused on the effect of finger-pointing alone rather than finger-pointing and calling. This was based on the previous findings demonstrating that (a) improved recognition of visual stimuli, including drug names, was shown when participants used finger-pointing alone (Mitobe \& Higuchi, 2016; Shinohara et al., 2008) and (b) finger-pointing can play a major role in anchoring people's eyes on a visual stimulus (Neggers \& Bekkering, 2000, 2001, 2002). The present study featured two hypotheses. The first was that nurses should recognize similar drug names quickly and accurately relative to non-medical participants. The second was that finger-pointing for each drug name reduces error due to the effect of anchoring people's eyes and focusing their attention on each drug name.

\section{Method}

\subsection{Participants and ethics statement}

Thirteen nurses (12 females, mean age $28.8 \pm 5.9$ years, experience $7.7 \pm 5.5$ years) and 15 graduate and undergraduate students (10 females, mean age $25.1 \pm 6.7$ years) participated. Nurses were recruited from the Nippon Medical School Tama Nagayama Hospital (Tokyo, Japan). Students were recruited from Tokyo Metropolitan University, Japan. All participants have normal or corrected-to-normal vision. The experiment protocol was approved by the institutional ethics committee of Tokyo Metropolitan University (24-2). Participants provided 
written informed consent to participate in this study. The tenets of the Declaration of Helsinki were followed.

\subsection{Apparatus}

Participants sat on a chair facing a laptop computer (LATITUDE-E5400, Dell, Inc.). A 14.1-inch LCD display was placed in front of them. A chinrest was used to maintain a distance of about $400 \mathrm{~mm}$ between the participants' eyes and the display. The experimental stimuli were presented using the stimulus presentation software SuperLab 4.0 (Cedrus, Inc.). Eye movement data were sampled using an eye-tracking system (EMR-9, Nac Image Technology, Inc.). The eye tracker, which was mounted on a hat, was a binocular corneal reflection system that measures the eye line of the gaze with respect to the temporal resolution of $30 \mathrm{~Hz}$. Frame-by-frame video-based analyses were performed using analysis software (EMR-dFactory, Nac Image Technology, Inc.) to identify where fixations were located.

\subsection{Stimulus}

The experimental target stimuli were drug names written in Katakana. The target stimuli were 38 drug names (36 for main trials and two for practice trials) that were frequently prescribed in the medical ward in the Nippon Medical School Tama Nagayama Hospital. All drug names consisted of five or six letters. We adopted actual drug names as a target and created pseudo names as similar and dissimilar distractors. For each of the 38 target stimuli, four similar and four dissimilar names were selected (see Table 1 for example).

Table 1. Six type of criteria for similar drug names

\begin{tabular}{|c|c|c|c|}
\hline & $\begin{array}{l}\text { Type of letter } \\
\text { modification }\end{array}$ & $\begin{array}{c}\text { Position of } \\
\text { letter modification }\end{array}$ & $\begin{array}{l}\text { Example target } \\
\text { ボルタレン }\end{array}$ \\
\hline $\mathrm{a}$ & \multirow{3}{*}{ substituted } & beginning & ギルタレン \\
\hline $\mathrm{b}$ & & middle & ボルクレン \\
\hline $\mathrm{c}$ & & end & ボルタルン \\
\hline d & \multirow{3}{*}{ transposed } & beginning - middle & ボタルレン \\
\hline e & & middle - end & ボルレタン \\
\hline $\mathrm{f}$ & & beginning - end & ボンタレル \\
\hline
\end{tabular}

Similar drug names were created using six criteria: (a) substitute a letter with another at the beginning of the target name, (b) substitute a letter with another in the middle of the name, (c) substitute a letter with another at the end of the name, (d) transpose each letter between the beginning and middle of the name, (e) transpose each letter between the middle and end of the name, (f) transpose each letter between the beginning and end of the name. Dissimilar drug names were randomly selected letters which not configured target names and placed the same string. Dissimilar drug names were created using randomly chosen letters that have the same letter length and was not included in the target name. 
The visual angle of each name presented on the display was $0.5^{\circ} \times 5.0^{\circ}$ for five-letter drug names and $0.5^{\circ} \times 5.7^{\circ}$ for six-letter drug names. The letters were presented in black on a white background.

\subsection{Task and procedure}

The task was a modified version of the task used in Mitobe and Higuchi (2016). We changed the following two points of the task. First, we adopted a six-choice reaction time task for drug names instead of a four-choice reaction time task in order to increase the difficulty. Second, we selected real drug names for targets and pseudo names for distractors, while real drug names were used for both targets and distractors in Mitobe and Higuchi. We used the pseudo names for distractors to strictly manipulate the degree of similarity to target names.

Prior to a main task, participants performed a practice task for memorizing predetermined key positions (a key-pressing task). The purpose of asking participants to perform this task was to let them become familiar with the six predetermined locations (two columns $\times$ three arrays). Familiarization with the task was necessary to remove the possibility that a delayed response observed in the main task could simply be due to be unfamiliarity with key locations. For the key-pressing task, each trial began with the presentation of a cross mark as a fixation location at the center of the display for $1000 \mathrm{~ms}$. One hundred milliseconds after the cross mark disappeared, five blackened squares appeared for $2000 \mathrm{~ms}$ at the same location. Then the fixation cross appeared again for $1000 \mathrm{~ms}$, and five squares were presented at six predetermined locations for $7000 \mathrm{~ms}$. In one of the six locations, the squares were solid black. Participants were asked to report the location of the black squares by pressing one of the six keys on a 10-key pad. In some trials black squares were not presented. In this case, the participants were instructed to press the "no" key on the pad when they determined there was no black square. The participants performed 21 trials for the task (18 trials for target presence and three trials for target absence).

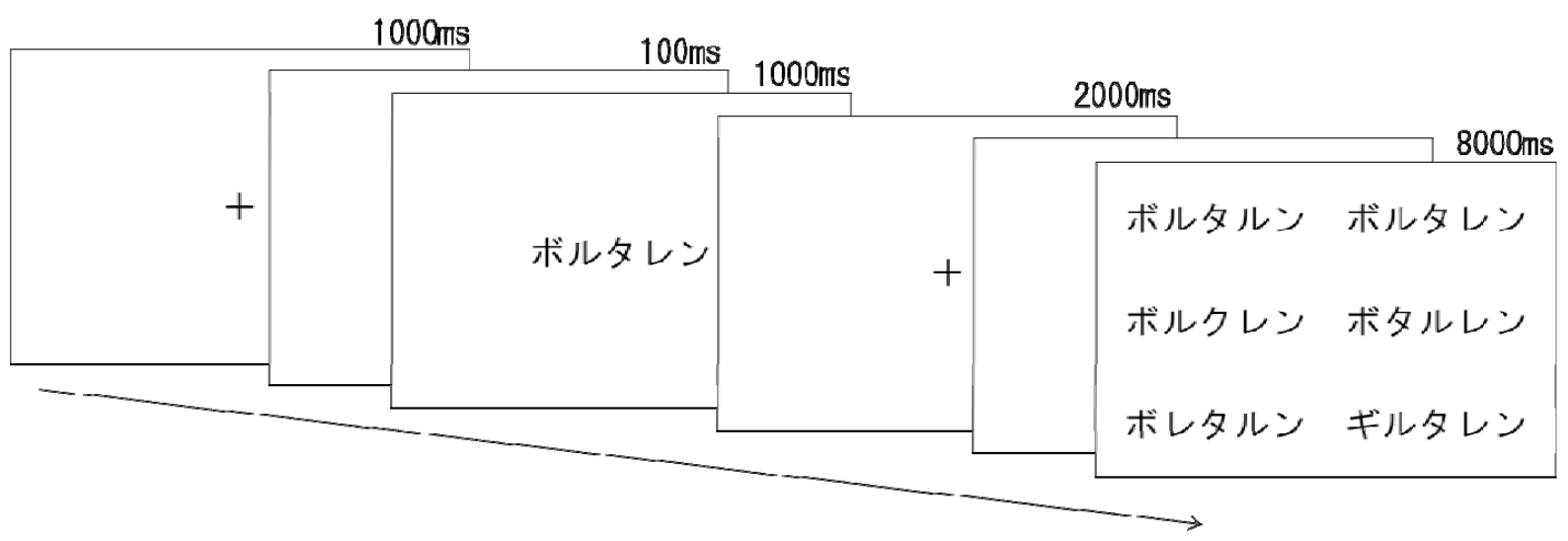

Figure 1. Example of presentation of stimulus

For the main task, each trial began with the presentation of a cross mark as a fixation location at the center of the display for $1000 \mathrm{~ms}$ (Figure 1). One hundred milliseconds after the cross disappeared, the target name was presented at the same location for $2000 \mathrm{~ms}$. Then the fixation cross was presented again for $1000 \mathrm{~ms}$. Six names were displayed for $10,000 \mathrm{~ms}$ in 
two columns $\times$ three arrays. Six drug names were constituted by including either of one, two, three, four, five or no similar names. To enhance the degree of task difficulty, the target was absent in 12.5 percent of the trials (total 36 trials). For the trials a similar drug name was presented instead of the target.

The participants were asked to look at each of the six names in a zigzag direction from upper-left to bottom-right as quickly as possible. They were instructed to search the names with or without finger-pointing. When finger-pointing was requested, the participants searched for a target by pointing just below the center of each drug name to avoid hiding the name with the finger. When finger-pointing was not requested, the participants' dominant hand was rested on their thigh. When they found the target, they specified the location of the name by pressing one of six predetermined keys on the 10-key pad. When they determined that the target was not present, they pressed the "no" key. The participants performed a total of 252 trials for the main task (two finger-pointing conditions $\times$ six conditions for a number of similar names $(5,4,3,2,1$, or 0$) \times 18$ repetitions and 36 no-target trials). The trials were divided into two blocks, depending on finger-pointing conditions. The order of blocks was counterbalanced, and a five-minute rest period was inserted between each block. A one-minute rest period was also inserted when the participants finished every 42 trials within each block (i.e., two times per block). Prior to the main trials, the participants performed 14 practice trials to familiarize themselves with the task before each block.

After performing the main task, participants in both occupation groups rated their knowledge about each of the 36 drug names used in the main task by choosing one of three options: (a) they know the drug's name and the effect, (b) they have heard the name but don't know the effect, and (c) they have never heard the name. Such rating was necessary to ensure that participants in the nurse group had sufficient knowledge about the drug names used in the study, while those in the student group did not.

\subsection{Data analysis}

The main dependent measures were the RT, the error rate, and the type of error. The RT was measured from the onset of the presentation of four drug names to the pressing of one of the predetermined keys. Both the RT and the error rate were statistically tested using a three-way (occupation, finger-pointing and the number of similar drug names) analysis of variance (ANOVA) with repeated measures of finger-pointing and the number of similar drug names. Given that the distribution of the error rate was not normal, we adjusted the error rate using the arcsine transformation for the statistical analysis. Partial eta-squared values ( $\eta$ 2) were calculated as an unbiased estimate of the effect size in an ANOVA.

To investigate the types of errors, we analyzed the error data on the basis of the two points. First, each response error was categorized as similar (i.e., participants chose a similar stimulus instead of the target), dissimilar, or no target (i.e., participants responded that there was no target in spite of target presence). The frequency of each category was calculated as a percentage. A Pearson's chi-squared test was conducted as a statistical test. Second, for the errors categorized as similar, we specified the type according to the six criteria, from (a) to (f), described in the "Stimuli" section. The frequency of each type was also calculated as a 
percentage. To statistically examine whether the difference in frequency of each type of error was significant between the groups, an occupation $\times$ type Pearson's chi-squared test was conducted for each type of error.

As a preliminary check to ensure that participants in the nurse group had sufficient knowledge about the drug names used in the study, while those in the student group did not, the participants' rating of their knowledge was quantified. When they chose (a) (they knew the drug's name and the effect), (b) (they have heard the name but did not know the effect), or (c) (they have never heard the name), the score was 2 points, 1 point, or 0 points, respectively. Therefore, the score was 72 when they knew all of the 36 drug names and their effects. A t-test was conducted to statistically compare the ratings between the nurse and student groups.

For all statistical tests, a $\mathrm{p}<.05$ value was accepted as statistically significant. For ANOVAs, significant the main effects and the interactions were further analyzed using Bonferroni-corrected pairwise comparisons.

\section{Results}

\subsection{Preliminary check: The number of known drug names}

The mean \pm standard deviation (SD) was $69.0 \pm 3.9$ points for nurses and $3.4 \pm 4.3$ points for students. The t-test showed that the score was significantly higher for the nurse group than for the student group $(t(25)=41.39, p<.001)$.

a) Nurse

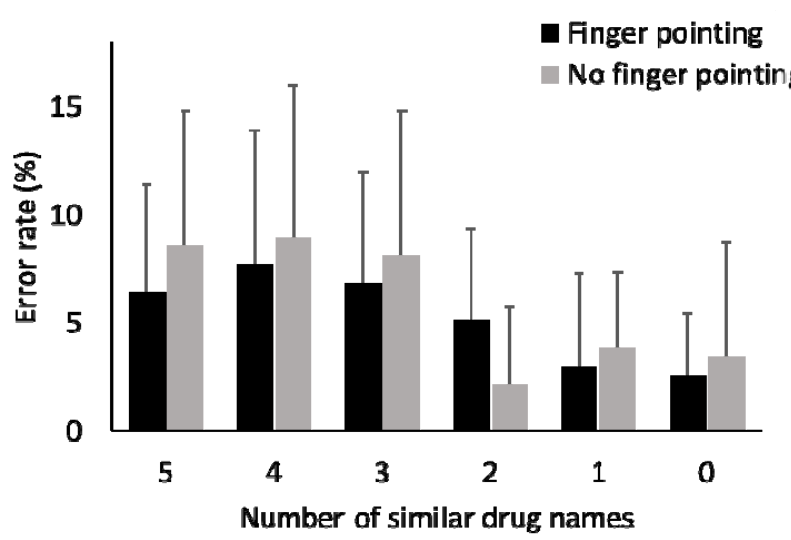

b)Adult

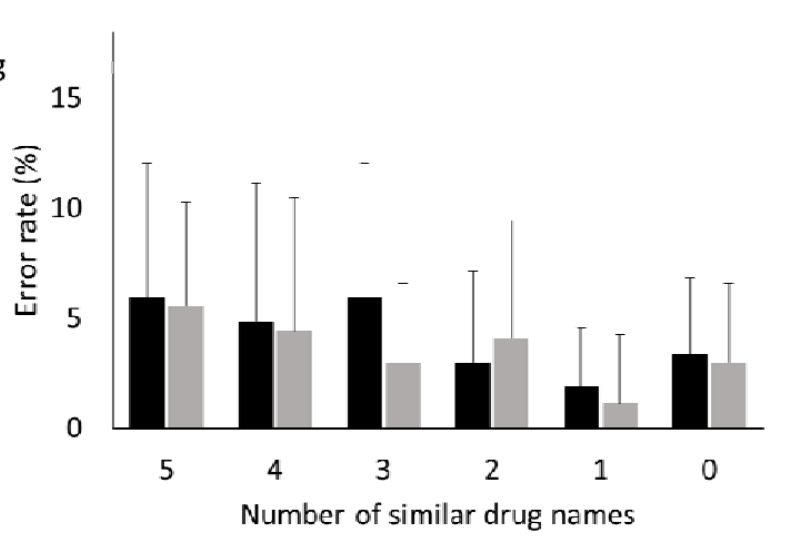

Figure 2. Error rate and standard deviation

\subsection{Error rate}

The means and SDs of the error rate under each condition are shown in Figure 2. A three-way ANOVA showed the main effect of occupation $(F(1,26)=4.6, p<.05, \eta p 2=.15)$. The error rate was significantly higher for the nurse group than for the student group. The main effect of the number of similar drug names was also significant $(F(5,130)=11.3, p<.001, \eta p 2$ $=.26$ ). Multiple comparisons with Bonferroni correction showed that the error rate was significantly higher with five, four or three similarities than with one or no similarity. 
Moreover, the error rate also was higher under the five similarity condition than the two similarity condition. Neither the main effect of finger-pointing $(F(1,26)=0.1$, n.s.), nor any two-way interactions (occupation $\times$ finger-pointing; $F(1,26)=0.19$, n.s.; occupation $\times$ the number of similar drug names, $\mathrm{F}(5,130)=1.8$, n.s.; or finger-pointing $\times$ the number of similarity, $\mathrm{F}(5,130)=0.6$, n.s. $)$, nor the three-way interaction $(\mathrm{F}(2,52)=1.4$, n.s. $)$ was significant.

\subsection{Reaction time}

The means and SDs of the RT under each condition are shown in Figure 3. The three-way ANOVA showed that the main effect of finger-pointing was marginally significant $(\mathrm{F}(1,26)$ $=4.2, \mathrm{p}=.054, \eta \mathrm{p} 2=.14)$. The main effect of the number of similar drug names was significant $(\mathrm{F}(5,130)=11.3, \mathrm{p}<.001, \eta \mathrm{p} 2=.30)$. Multiple comparisons with Bonferroni correction showed that the RT became significantly longer as the number of similar drug names increased, except for the pair of five and four similarities. The main effect of occupation was not significant $(\mathrm{F}(1,26)=2.6$, n.s. $)$. There was a marginally significant interaction between the finger-pointing and the number of similar drug names $(\mathrm{F}(1,26)=4.3$, $\mathrm{p}<.057, \eta \mathrm{p} 2=.14$ ). Any other interactions were not significant (occupation $\times$ finger-pointing, $\mathrm{F}(1,26)=1.1$, n.s.; occupation $\times$ the number of similar drug name, $\mathrm{F}(5,130)$ $=2.2$, n.s.; occupation $\times$ finger-pointing $\times$ the number of similarity, $\mathrm{F}(5,130)=0.6$, n.s. $)$.

a) Nurse

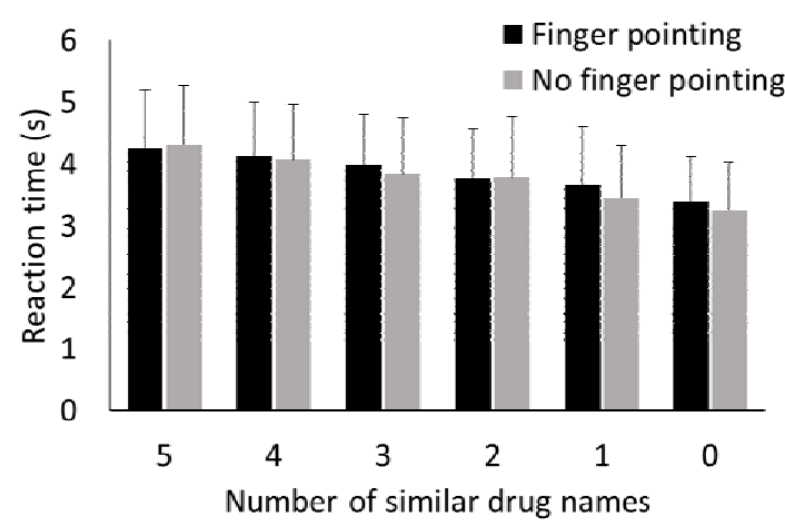

b)Adult

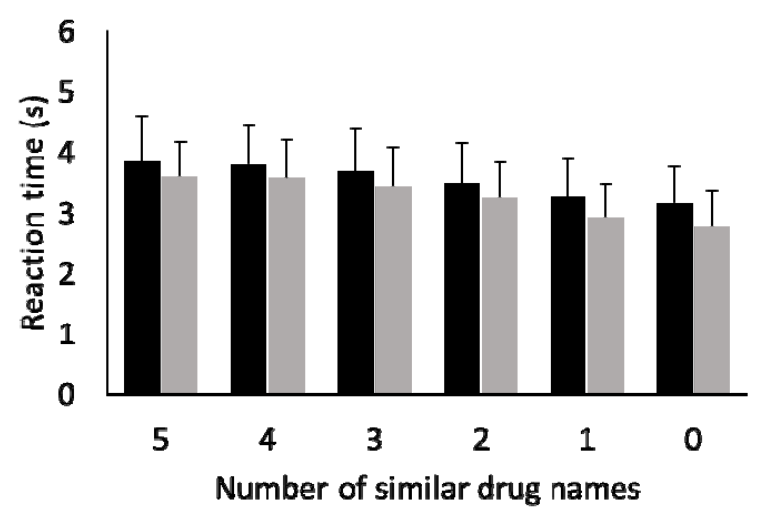

Figure 3. Reaction time and standard deviation

Table 2. Error type analysis about error trials

\begin{tabular}{|c|c|c|c|c|c|}
\hline & $\begin{array}{c}\text { Type of } \\
\text { letter modification }\end{array}$ & $\begin{array}{c}\text { Position of } \\
\text { letter modification }\end{array}$ & Nurse $(\%)$ & Student $(\%)$ & Total \\
\hline $\mathrm{a}$ & \multirow{3}{*}{ substituted } & beginning & $6(6.3)$ & $10(13.5)$ & 16 \\
\hline $\mathrm{b}$ & & middle & $7(7.3)$ & $2(2.7)$ & 9 \\
\hline $\mathrm{c}$ & & end & $21(21.9)$ & $16(21.6)$ & 37 \\
\hline d & \multirow{3}{*}{ transposed } & beginning - middle & $25(26.0)$ & $23(31.1)$ & 48 \\
\hline $\mathrm{e}$ & & middle - end & $35(36.5)$ & $16(21.6)$ & 51 \\
\hline $\mathrm{f}$ & & beginning - end & $2(2.1)$ & $7(9.5)$ & 9 \\
\hline
\end{tabular}




\subsection{Analysis of error type}

The total number of errors was 157 for the nurse group and 124 for the student group. The number of errors categorized as similar, dissimilar, or no target was $96(61.1 \%), 23(14.6 \%)$, and $38(24.2 \%)$ for nurses and $74(59.7 \%), 17(13.7 \%)$, and $33(26.6 \%)$ for students, respectively. An occupation $\times$ type Pearson's chi-squared test found no significant differences in error type between participant groups in any of three error types. Moreover, as Table 2 shows, we classified errors with similar drug names into six types using the criteria of experimental stimuli. The chi-squared test conducted for the categorized data showed that, for criteria (e), when there was a distractor that transposed each letter between the middle and end of the name, the difference between the nurse and student groups was significant $(\chi 2$ (5) $=11.74, \mathrm{p}<.05)$. Participants in the nurse group made a mistake more often than those in the student group.

\section{Discussion}

The purpose of this study was to investigate (a) whether nurses, as experts in medication, would be able to recognize similar drug names accurately when rapid reaction was requested and (b) whether nurses would benefit from finger-pointing. The results showed that the error rates were significantly higher for nurses than for students. Because the RT was comparable between the nurse and control groups, the higher error rates in the nurse group were not simply due to a speed-accuracy trade-off (i.e., nurses made more errors when aiming for a rapid reaction). Moreover, nurses did not benefit from finger-pointing.

\subsection{Likelihood of nurse error}

In the present study, the nurses, who are experts in medication, showed the difficulty distinguishing between similar drug names. Drug names are generally written in Katakana, which is a phonogram and meaningless in itself. People who are not familiar with medication seem to recognize the letter strings of the drug name as a nonsense syllable. In contrast, the nurses recognize it as a meaningful word and, consequently, the top-down process becomes dominant. Recognition of words tends to become confused when these words are different due to the transposition and/or substitution of the letters (Perea \& Lupker, 2003; Grainger \& Whitney, 2004). Because the stimuli of drug names in the present study were created so that they transposed each letter between the middle and end of the drug name, the confusion errors are likely to occur more frequently for the nurses who would misrecognize the stimuli as meaningful words.

The analysis of error type showed that both the nurses and students made more errors with similar drug names as a target than with dissimilar drug names. In particular, the nurses tended to misperceive similar names in which two letters between the middle and end of the names were transposed. Previous studies showed that pseudo words in which two letters are transposed are more likely to be confusing than pseudo words in which a letter is substituted with another letter (O'Connor \& Foster, 1981; Perea \& Lupker, 2004). When the similar name in which two letters between the middle and end of the name are transposed is presented with the target name, these two names are the same at the beginning; this could 
lead to a top-down process for familiar words and would tend to result in the confusion errors.

\subsection{Effect of error prevention by finger-pointing}

In the present study, we failed to find the effect of decreasing the error rate by finger-pointing. There was not much difference in the error rate between the finger-pointing conditions. This is inconsistent with Mitobe and Higuchi (2016), who demonstrated that finger-pointing was effective to reduce the error rate when a single similar word existed. A possible explanation for this discrepancy would be the difference in drug names used as experimental stimuli. In the present experiment, only target stimuli were existing drug names, whereas distractors were pseudo names (i.e., some letters were transposed or substituted). In Mitobe and Higuchi, both targets and distractors were existing drug names. Pseudo names were used in the present study to strictly control the similarity between the target and distractor. The use of pseudo names as distractors may have increased the cognitive load to search for the target name; as a result, the possible effect of finger-pointing may not have been sufficient to decrease the cognitive load.

In the earlier work using a choice reaction task that imitated traffic lights (Haga et al., 1996), finger-pointing and calling were effective for error prevention, and a similar effect was found for finger-pointing alone. Moreover, Masuda et al. (2014) showed that the error rate decreased with finger-pointing in a counting task, a go/no go task, and task switching. The stimuli in these experiments were relatively simple objects such as color symbols of traffic lights, dots, or numbers, while the stimuli in this study were drug names. Drug names are letter strings that are more complex than symbols and numbers. The results may suggest that the effect of finger-pointing is limited for such complex stimuli. In addition, because nurses are very familiar with drug names, the process of stimulus recognition may have involved lexical and semantic analyses. It is possible that finger-pointing may not be as effective in situation where a higher order of cognitive function comes into play.

In the present study, we focused on the effect of finger-pointing alone rather than finger-pointing and calling. Shinohara, Naito, Matsui, and Hikono (2012) explained that finger-pointing acts on visual systems, directing observers' eyes and focus of attention, while calling acts on the central executive function of working memory, selecting and activating appropriate information. Masuda et al. (2014) also showed that calling affects working memory and facilitate memory. It is possible that repeated calling of the target drug names reinforces memory and facilitates the detection of differences between the target and similar drug names. Future studies are necessary to investigate this.

\section{Conclusion}

The present study showed that nurses made errors in discrimination between similar drug names more frequently when rapid reaction was requested. We propose that this rather unexpected result may have occurred because the nurses' familiarity with medicines facilitates top-down processing, which results in inhibition of detecting the difference between the target drug names and similar names. In addition, finger-pointing was inadequate 
to prevent error with complex objects such as drug names, even if it is effective with simple objects such as symbols or signals.

\section{Acknowledgements}

I would like to Kazunobu Fukuhara for his helpful and constructive comments.

\section{References}

Berman, A. (2004). Reducing medication errors through naming, labeling, and packaging. Journal of Medical Systems, 28, 9-29. https://doi.org/10.1023/B:JOMS.0000021518.60670. 10

Carey, D. P. (2000). Eye-hand coordination: Eye to hand or hand to eye? Current Biology, 10, 416-419. https://doi.org/10.1016/S0960-9822(00)00508-X

Deconinck, F.J. A., Van Polanen, V., Savelsbergh, G. J. P., \& Bennett, S. J. (2011). The relative timing between eye and hand in rapid sequential pointing is affected by time pressure, but not by advance knowledge. Experimental Brain Research, 213, 11-109. https://doi.org/10.1007/s00221-011-2782-0

Grainger, J. \& Whitney, C. (2004). Does the huamn mnid raed wrods as a wlohe? TRENDS in Cognitive Sciences, 8, 58-59. https://doi.org/10.1016/j.tics.2003.11.006

Haga, S., Akatsuka, H., \& Shirato, H. (1996). Laboratory experiments for verifying the effectiveness of "finger-pointing and call" as a practical tool of human error prevention. Japanese Association of Industrial/ Organizational Psychology Journal, 9, 107-114 (In Japanese with English abstract).

Japan Council for Quality Health Care Division of Adverse Event Prevention (2016). II. Current Reporting Status. Project to Collect Medical Near-miss/ Adverse Event Information 2015 Annual Report. 55-75.

Kawamura, H. (2003). Complete error map from 11,000 cases of medical incidents. Tokyo: Igaku-Shoin (in Japanese, translated by the author of this article).

Kohn, L., Corrigan, J., \& Donaldson, M. (1999). To err is human: Building a safer health system. Washington DC, Institute of Medicine. https://doi.org/10.17226/9728

Lambert, B. L., Chang, K.Y., \& Gupta, P. (2003). Effects of frequency and similarity neighborhoods on pharmacists' visual perception of drug names. Social Science \& Medicine, 57, 1939-1955. https://doi.org/10.1016/S0277-9536(03)00059-5

Lambert, B. L., Chang, K.Y., \& Lin, S. J. (2001). Effect of orthographic and phonological similarity on false recognition of drug names. Social Science \& Medicine, 52, 1843-1857. https://doi.org/10.1016/S0277-9536(00)00301-4

Lambert, B. L., Chang, K.Y., \& Lin, S. J. (2003). Immediate free recall of drug names: Effects of similarity and availability. American Journal of Health System Pharmacy, 60, 156-169. 
Masuda, T., Shigemori, M., Sato, A., \& Haga, S. (2014). Error prevention effects of point and call checks. RTRI REPORT, 28, 5-10 (in Japanese with English abstract).

McClelland, J.L., \& Rumelhart, D. E. (1981). An interactive activation model of context effects in letter perception: Part1. An account of basic findings. Psychological Review, 88, 375-407. http://psycnet.apa.org/doi/10.1037/0033-295X.88.5.375

Mitobe. J. \& Higuchi, T. (2016). Does finger-pointing toward drug names prevent confusion between similar drug names during rapid search? Psychologia, 59, 38-49. https://doi.org/10.2117/psysoc.2016.38

Nabeta, K., Imai, T., Kimura, M., Ohkura, M., \& Tsuchiya, F. (2011). The similarity index of medicine names based on character shape similarity. Transactions of Japan Society of Kansei Engineering, 10, 287-294 (in Japanese with English abstract).

Neggers, S. F. W., \& Bekkering, H. (2000). Ocular gaze is anchored to the target of an ongoing pointing movement. Journal of Neurophysiology. 83, 639-651. https://doi.org/10.1152/jn.2000.83.2.639

Neggers, S. F. W., \& Bekkering, H. (2001). Gaze anchoring to a pointing target is present during the entire pointing movement and is driven by a non-visual signal. Journal of Neurophysiology, 86, 961-970. https://doi.org/10.1152/jn.2001.86.2.961

Neggers, S. F. W., \& Bekkering, H. (2002). Coordinated control of eye and hand movements in dynamic reaching. Human Movement Science, 21, 349-376. https://doi.org/10.1016/S0167-9457(02)00120-3

O’Connor, R.E. \& Forster, K.I. (1981). Criterion bias and search sequence bias in word recognition. Memory \& Cognition, 9, 78-92. https://doi.org/10.3758/BF03196953

Perea, M. \& Lupker, S. J. (2003). Does jugde activate COURT? Transposed-letter similarity effects in masked associative priming. Memory \& Cognition, 31, 829-841. https://doi.org/10.3758/BF03196438

Perea, M.\& Lupker, S. J. (2004). Can CANISO activate CASINO? Transposed-letter similarity effects with nonadjacent letter positions. Journal of Memory and Language, 51, 231-246. https://doi.org/10.1016/j.jml.2004.05.005

Pham, J. C., Aswani, M. S., Rosen, M., Lee, H. W., Huddle, M., Weeks, K., \& Pronovost, P. J. (2012). Reducing medical errors and adverse events. Annual Review of Medicine, 63, 447-463. https://doi.org/10.1146/annurev-med-061410-121352

Posner, M. I. (1980). Orienting of attention. The Quarterly Journal of Experimental Psychology, 32, 3-25. http://dx.doi.org/10.1080/00335558008248231

Rumelhart, D. E., \& McClelland, J. L. (1982). An interactive activation model of context effects in letter perception: Part2. The contextual enhancement effect and some tests and extension of the model. Psychological Review, 89, 60-94. http://psycnet.apa.org/doi/10.1037/0033-295X.89.1.60 


\section{Macrothink}

Shinohara, K., Morimoto, K., \& Kubota, T. (2009). The effect of "Finger-pointing and Call" on orientation of visual attention. The Japan Journal of Ergonomics, 45, $54-57$ (In Japanese). https://doi.org/10.5100/jje.45.54

Shinohara, K., Naito, H., Matsui, Y., \& Hikono, M. (2012). The effects of " finger-pointing and calling" on cognitive control processes in the task-switching paradigm. International Journal of Industrial Ergonomics, 43, 129-136. https://doi.org/10.1016/j.ergon.2012.08.004

Suzuki, S., Shimizu, H., Tsuchiya, F. Kasamatsu, K., Yamanaka, K., \& Kawakami, M. (2005). A survey research on the medication error: An investigation about the relationship between the similarity of names of medicines and the occurrence of medication errors. Japan Journal of Physiological Anthropology, 10, 145-152 (in Japanese with English abstract). https://doi.org/10.20718/jjpa.10.4_145

Tsuchiya, F., Kawamura, N., Oh, C., \& Hara, A. (2001). Standardization and similarity deliberation of drug-names. Japan Journal of Medical Informatics, 21, 59-67 (in Japanese with English abstract). https://doi.org/10.14948/jami.21.59

Yamade, Y., Haga, S., Tsuchiya, F., \& Shin, H. S. (2006). Similarity of drug names and confusion errors; Laboratory experiments with students and pharmacists. Cognitive Studies, 13, 80-95 (in Japanese with English abstract). https://doi.org/10.11225/jcss. 13.80

\section{Copyrights}

Copyright for this article is retained by the author(s), with first publication rights granted to the journal.

This is an open-access article distributed under the terms and conditions of the Creative Commons Attribution license (http://creativecommons.org/licenses/by/4.0/) 